\title{
A new function of glucocorticoid receptor: regulation of mRNA stability
}

\author{
Ok Hyun Park, Eunjin Do \& Yoon Ki Kim* \\ Division of Life Sciences, Korea University, Seoul 136-701, Korea
}

\begin{abstract}
It has long been thought that glucocorticoid receptor (GR) functions as a DNA-binding transcription factor in response to its ligand (a glucocorticoid) and thus regulates various cellular and physiological processes. It is also known that GR can bind not only to DNA but also to mRNA; this observation points to the possible role of GR in mRNA metabolism. Recent data revealed a molecular mechanism by which binding of GR to target mRNA elicits rapid mRNA degradation. GR binds to specific RNA sequences regardless of the presence of a ligand. In the presence of a ligand, however, the mRNA-associated GR can recruit PNRC2 and UPF1, both of which are specific factors involved in nonsense-mediated mRNA decay (NMD). PNRC2 then recruits the decapping complex, consequently promoting mRNA degradation. This mode of mRNA decay is termed "GR-mediated mRNA decay" (GMD). Further research demonstrated that GMD plays a critical role in chemotaxis of immune cells by targeting CCL2 mRNA. All these observations provide molecular insights into a previously unappreciated function of GR in posttranscriptional regulation of gene expression. [BMB Reports 2015; 48(7): 367-368]
\end{abstract}

Glucocorticoid receptor (GR) is one of typical nuclear receptors and is known to function as a transcription factor. In the absence of a glucocorticoid (the ligand of GR), GR is mostly located in the cytoplasm. In the presence of a glucocorticoid

*Corresponding author. E-mail: yk-kim@korea.ac.kr

http://dx.doi.org/10.5483/BMBRep.2015.48.7.131

Received 2 July 2015

Keywords: Glucocorticoid receptor, mRNA decay, PNRC2, UPF1, Chemotaxis

Abbreviations: GR, Glucocorticoid receptor; GMD, GR-mediated mRNA decay; UPF1, upstream frames-shift 1; PNRC2, Proline-rich nuclear receptor coregulatory protein 2; GMD, Glucocorticoid receptor--mediated mRNA decay; NMD, nonsense-mediated mRNA decay

Perspective to: Hana Cho et al (2015), Glucocorticoid receptor interacts with PNRC2 in a ligand-dependent manner to recruit UPF1 for rapid mRNA degradation. Proc. Natl. Acad. Sci. USA, 112(13): E1540-E1549. (which reached the cytosol by crossing the plasma membrane), the cytosolic GR binds to the glucocorticoid and the resulting glucocorticoid-GR complex relocates to the nucleus, dimerizes, and then binds to specific cis-acting elements along with coregulatory proteins; this event leads to either transcriptional activation or repression. According to a recent report, in addition to $\mathrm{GR}^{\prime}$ s role in transcriptional regulation, this protein participates in regulation of mRNA stability as an RNA-binding protein; however, the underlying molecular mechanism remained unclear. Our recent study showed a new mode of gene regulation whereby GR triggers rapid mRNA degradation in a ligand-dependent manner; we termed this mechanism "GR-mediated mRNA decay" (GMD).

For efficient mRNA degradation, GMD requires a ligand, PNRC2, and UPF1. Immunoprecipitation (IP) experiments showed that treatment of cells with dexamethasone, which is a potent synthetic glucocorticoid, promotes formation of a protein complex containing GR, PNRC2, UPF1, and DCP1A (a component of the decapping complex). It is known that (i) GR directly interacts with PNRC2 and (ii) PNRC2 directly interacts with UPF1 and DCP1A; therefore, PNRC2 may serve as a link for the association of GR with UPF1 and of GR with DCP1A. These molecular events were clearly demonstrated in IP experiments showing that downregulation of endogenous PNRC2 by means of a specific small interfering RNA (siRNA) destabilizes the GR-UPF1 and GR-DCP1A interactions. In addition, downregulation of endogenous UPF1 destabilizes the association between GR and PNRC2, indicating that UPF1 further stabilizes the interaction between GR and PNRC2 in the presence of a ligand.

A search for cellular GMD substrates by microarray analysis yielded four putative substrates: mRNAs of B-Cell CLL/ymphoma 3 (BCL3); zinc finger SWIM-type containing 4 (ZSWIM4); pleckstrin homology-like domain, family $A$, member 1 (PHLDA1); and a previously reported GMD substrate: the mRNA of chemokine (C-C motif) ligand 2 (CCL2). The abundance and half-life of all these putative GMD substrates were reduced when cells were treated with Dex but were significantly restored when the cells were depleted of GR, PNRC2, or UPF1 by means of specific siRNAs. Furthermore, a series of experiments involving chimeric reporter constructs showed that insertion of a GR-binding site, which is present in the GMD substrates, into either the 5' untranslated region (5'UTR) 


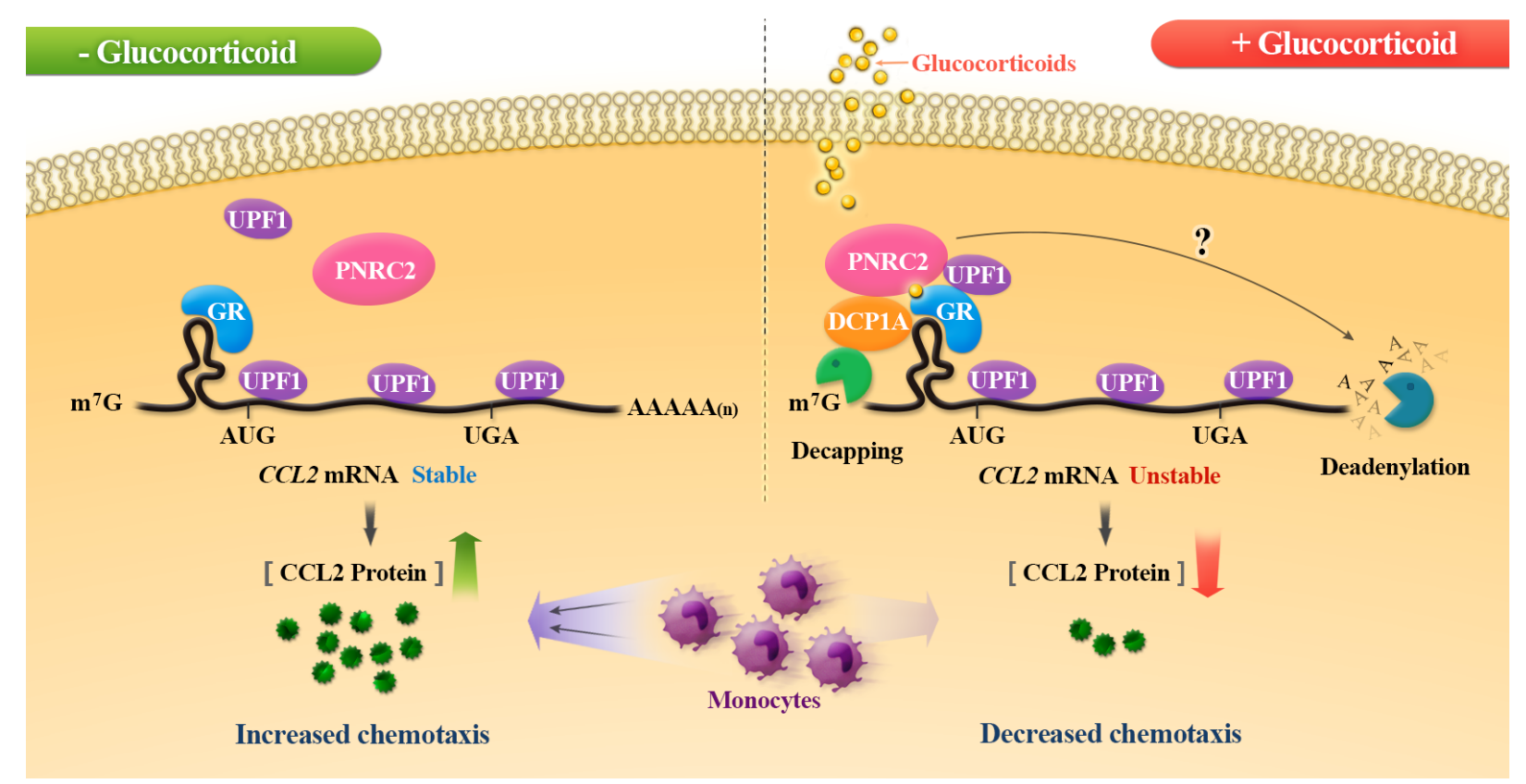

Fig. 1. A model of the regulation of chemotaxis by glucocorticoid receptor (GR)-mediated mRNA decay (GMD). (left) Because GMD requires a ligand, GMD is inefficient in the absence of a glucocorticoid and consequently CCL2 mRNA is not degraded rapidly. Thus, the CCL2 protein that is synthesized from stable CCL2 mRNA increases the efficiency of chemotaxis of monocytes. (right) In the presence of a glucocorticoid, GMD is efficient and destabilizes CCL2 mRNA. Consequently, the level of the CCL2 protein is reduced and chemoattraction is inhibited.

or 3'UTR of a reporter mRNA triggers efficient degradation of the reporter mRNA. These results indicate that loading of GR onto a GR-binding site within a target mRNA is sufficient for effective GMD. In support of this notion, artificial tethering of GR to the 5'UTR of a reporter mRNA by means of the $\lambda N-5 B o x B$ system elicits rapid mRNA degradation in the presence of a ligand in a PNRC2- and UPF1-dependent manner.

The CCL2 protein, a member of the CC chemokine family, is involved in chemotaxis via which a variety of immune cells are recruited to sites of inflammation. Because CCL2 mRNA is a GMD substrate, CCL2-mediated chemotaxis of monocytes is expected to be affected by GMD. Indeed, experiments with a chemotaxis chamber revealed that chemotaxis of the human acute monocytic leukemia cell line THP-1, which expresses CCL2 receptors on the plasma membrane, is affected by GMD in the donor cells that secrete CCL2 (Fig. 1).

Our findings about GMD raise many interesting questions. PNRC2 and UPF1, both of which were found to be GMD factors, had originally been identified as factors involved in nonsense-mediated mRNA decay (NMD), via which abnormal mRNAs harboring a premature termination codon are selectively degraded. In the case of NMD, UPF1 joins a ribosome terminating on a premature termination codon and then triggers NMD. In other words, UPF1 is recruited to the NMD machinery in a translation-dependent manner. In the case of GMD, however, UPF1 recruitment turned out to be independent of a translation event. UPF1 stabilizes the GR-containing complex and its helicase function is necessary for GMD; however, the translation-independent recruitment of UPF1 and consequent mRNA degradation challenge the widely accepted model of UPF1 function in mRNA degradation. Another important question is whether other nuclear receptors can bind to RNA as GR does. Because PNRC2 can interact with many types of nuclear receptors, other nuclear receptors that are loaded onto an mRNA may recruit PNRC2 and PNRC2-interacting proteins (UPF1 and DCP1A) and thereby may elicit rapid mRNA degradation. Future studies on these topics will improve the understanding of nuclear receptor-mediated mRNA decay and its biological relevance.

\section{ACKNOWLEDGEMENTS}

This work was supported by the National Research Foundation of Korea (NRF) grant funded by the Korea government (MSIP) (2012R1A2A1A01002469 and 2014R1A2A1A11050412), and by a Korea University Grant (K1505301). O.H.P was in part supported by the Global PhD Fellowship Program through the National Research Foundation funded by the Korean Government. 